\title{
Evolution of Processor Architecture in Mobile Phones
}

\author{
Mahendra Pratap Singh \\ Research Scholar, \\ Department of Computer Science, Mohanlal \\ Sukhadia University, Udaipur, India
}

\author{
Manoj Kumar Jain, Ph. D \\ Associate Professor, Computer Science \\ Mohanlal Sukhadia University \\ Udaipur, India
}

\begin{abstract}
Mobile phone has become a vital component of our daily life. Technological advancements have resulted in significant changes in the processor architecture of mobile phones; transforming the typical mobile phones of 1990's to modern smart phones. The design and deployment of mobile processors over the years is largely affected by Communication, performance, and low-power operation. The transition from analog to digital telephony has resulted in mobile devices delivering a wide range of data services. To support these services, processor architecture has now become much more complex. Mobile processors are growing rapidly with each passing generation. The goal of this paper is to review various processor architectures for mobile phones.
\end{abstract}

\section{Keywords}

Processor architecture, DSP, VLIW, SoC, ARM processors

\section{INTRODUCTION}

Smartphone hardware mainly consists of application processors (System-on-a-Chip), RAM (mobile SDRAM/ mobile DDR), DSP, CPU (ARM processor), etc. According to Farinaz et al [1], Processors that are used for mobile phones are subject to design metrics that emphasize cost, time-tomarket, and low power. Because of the constrained resources of power and cost, and the real-time computation requirements, the processors for use in mobile applications possess a number of distinct characteristics such as limited programmability.

Processor architecture of mobile devices delivering data services must provide support for much more complex user interface, dynamic operating environments, and support for additional services. To provide for these additional requirements, advanced architectures may include multiple DSP's or hardware coprocessors.

\section{MOBILE PROCESSOR ARCHITECTURE TRENDS 2.1 Traditional DSP (Digital Signal Processor) Architectures}

There have been two distinct approaches for implementation of cellular handsets. One approach emphasizes programmable DSP's, while the other approach utilizes ASIC (Applicationspecific integrated circuit) techniques [6].

DSP is a specialized microprocessor used for mobile phones. Historically DSP's were designed around single stand-alone integrated circuits (ICs). Embedded DSP's are now widely adopted for VLSI designs. Programmable DSP's are dominant in wireless handset market for digital cellular telephony.

First generation of mobile communications i.e. $1 \mathrm{G}$ systems used analog transmission with the limitations of requiring more power for transmission and allowing limited users[2].

Global System for Mobile Communications (GSM) standard evolved after first generation of mobile communication for analog cellular networks. DSP processors form one of the most important classes of mobile embedded processors in second generation i.e. $2 \mathrm{G}$ systems.

DSP architectures are preferred over ASIC due to shorter product life cycle and they are extensively used in GSM mobiles. Programmable DSP's provide a cost-effective and flexible architecture for cellular telephones. AT\&T in 1979 introduced first DSP, and subsequently Texas Instruments came up with other DSP's.

Traditionally, DSP used Harvard architecture which physically separates storage and signal pathway for instructions and data [2]. This is in contrast with Von Neumann Architecture, where data and instructions are stored in the same memory. As shown in Figure 1, it requires data memory and instruction memory to execute instructions. It has separate data and instruction buses allowing simultaneous transmission. Subsequently, the output of multiplication unit connects to an adder, thereby adding and saving all partial results for further processing.

This architecture results in fewer cycles for executing a particular function as it enables high memory bandwidth and multiple operand operations. Multiply-accumulate (MAC) instructions are commonly associated with DSP architectures. 


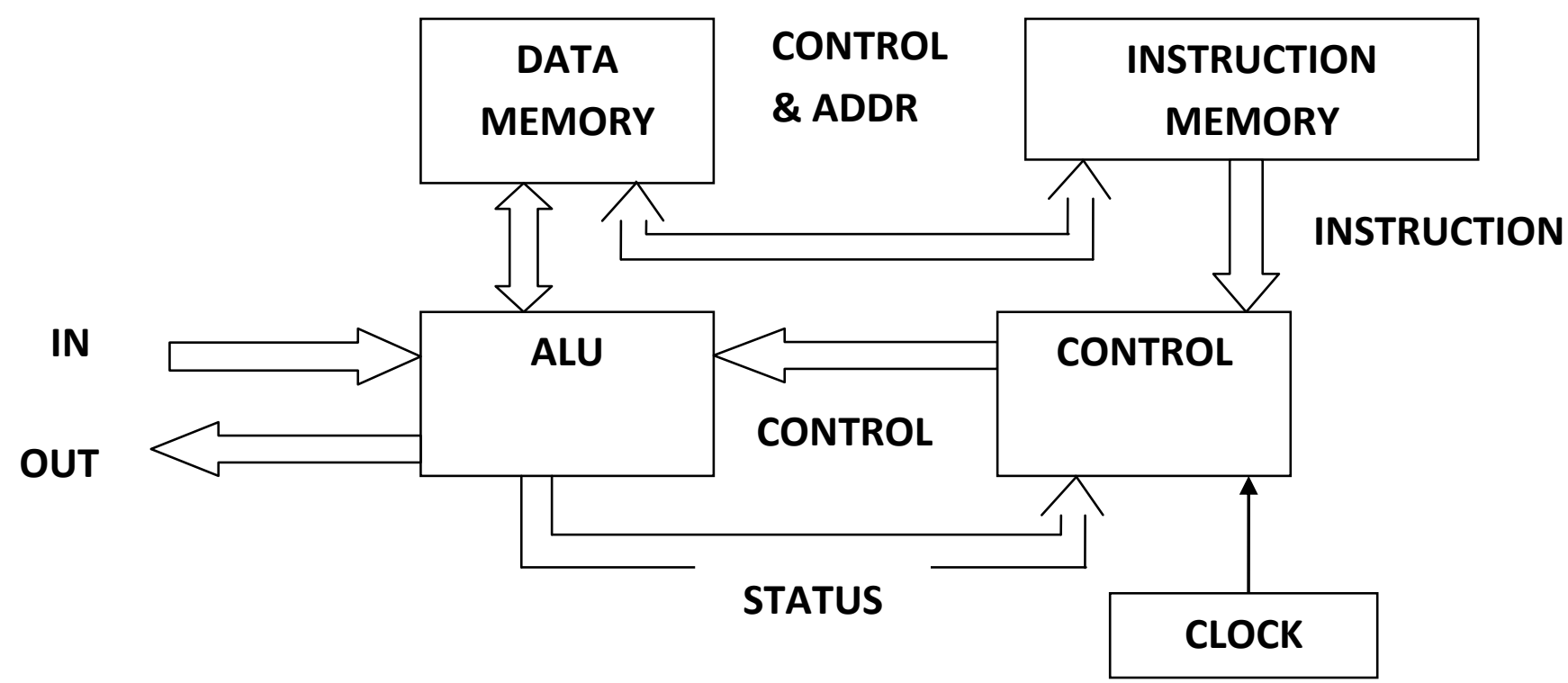

Figure 1: Traditional DSP Architecture (Harvard Architecture)

\subsection{Modern DSP Architectures}

Apart from traditional architectures, some modern DSP architectures have evolved for mobile devices. TMS320C55 is a modern DSP architecture which implements Harvard architecture utilizing one and three read buses for code and data, respectively [2]. With TMS320C55 DSP architecture, features like programmable idle modes and automatic power saving were incorporated for better processor utilization at top speeds.

TMS320C62XX is an example of VLIW (Very long instruction word) DSP processor. VLIW architecture in DSP provides a compiler based programmer friendly environment. These VLIW processors follow explicitly parallel instruction computing (EPIC) architectures.

The TigerSHARK DSP architecture has a series of advanced features like the use of "short vectors" to process information using SIMD (single instruction multiple memory) architecture [2].

DSPs have become common in mobile devices because they provide real-time operation at low power costs. Future mobile devices have to be aligned to integrate more functions, considering computational power requirements. Advancements in DSP lead to higher clock frequencies as well as a reduced power consumption per MIPS for mobile phones [3].

One way of customization is parallelism in superscalar designs, which is widely implemented for future mobile processors. VLIW and SIMD architectures in modern cellular devices are becoming more and more popular; because they allow reducing the frequency and voltage of the CPU chips without losing performance. Modern DSPs can be more effective if they are able to support parallel processing.

In modern DSP architectures, computational power has greatly improved due to advancements in chip fabrication. As shown in Table 1 below, the same DSP chip was providing approximately 5 GIPS (Giga Instructions per Second) in 2000, in contrast to 5 MIPS in 1980 and it has grown to 50 GIPS in the year 2010. Considering other factors also, the advancements in DSP integration is significant.
Table 1: DSP Integration over the Years

\begin{tabular}{|l|c|c|c|c|}
\hline Parameter & $\mathbf{1 9 8 0}$ & $\mathbf{1 9 9 0}$ & $\mathbf{2 0 0 0}$ & $\mathbf{2 0 1 0}$ \\
\hline Die Size (mm) & 50 & 50 & 50 & 5 \\
\hline $\begin{array}{l}\text { Technology } \\
\text { (micrometers) }\end{array}$ & 3 & 0.8 & 0.1 & 0.02 \\
\hline $\mathrm{MIPS}$ & 5 & 40 & 5000 & 50000 \\
\hline $\mathrm{MHz}$ & 20 & 80 & 1000 & 10000 \\
\hline RAM (bytes) & 256 & 2000 & 32000 & 1000000 \\
\hline Price (dollars) & 150 & 15 & 5 & 0.15 \\
\hline $\begin{array}{l}\text { Power } \\
\text { (mW/MIPS) }\end{array}$ & 250 & 12.5 & 0.1 & 0.001 \\
\hline $\begin{array}{l}\text { Transistors } \\
\text { Wafer Size }\end{array}$ & 50000 & 500000 & $\begin{array}{c}5 \\
\text { million }\end{array}$ & $\begin{array}{c}50 \\
\text { million }\end{array}$ \\
\hline \begin{tabular}{l} 
(inches) \\
\hline
\end{tabular} & 6 & 12 & 12 \\
\hline
\end{tabular}

Source: Past and Projected Evolution of DSP [Gene 2000)

A number of FPGA (Field-programmable gate array) architectures have been specifically designed for DSP to effectively utilize VLSI resources. VLIW compilers are extensively used in DSP architectures to support instruction level parallelism [. The trend in modern DSP architectures is to develop fault-tolerant and reliable DSP systems. DSP systems are reconfigured keeping in mind a number of design goals like performance, power, reliability, cost, and development time. Modern DSP systems are implemented based on reconfigurable DSP architectures.

In modern DSP's, architecture can be extended by duplicating the processor cores. Enhanced DSP's utilizes SIMD operations, while multiple-issue DSP's may implement either VLIW or superscalar architectures.

\subsection{System on Chip (SoC) based architectures}

Mobile device processor architecture became simple with SOC designs. Real time responsiveness in mobile devices can be managed by using an enhanced DSP hybrid chip. Lowering the voltage of the chip enables low power operation in mobile devices. 
Matthias et al [5] proposed a new scalable DSP architecture for SoC domains. In SoC based designs, system tasks can be managed by integrating microcontrollers, dedicated ASIC's, or DSP's in a single chip as shown in Figure 2 below:

Figure 2: Traditional DSP Architecture (Harvard Architecture)

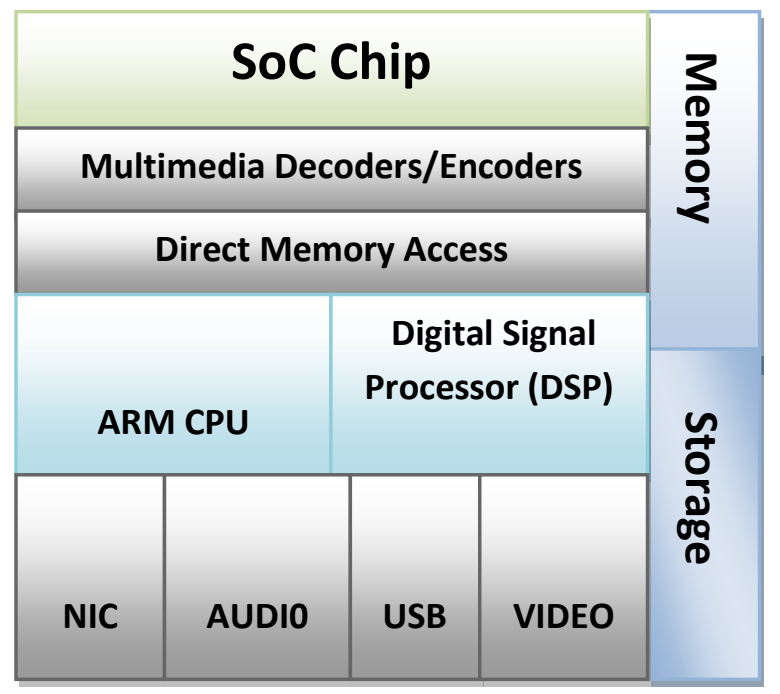

Highly integrated SoC's leveraging multicore technology has emerged for higher performance and low power designs. Low power operation often limits the architectural choices. High throughput of VLIW architectures in mobile devices requires a fast memory system like cache memories.

To speed up the operation of mobile devices, instruction se customizations have been done by many companies.ARM Ltd. has done extensive instruction set customization by encoding most used instructions in 16-bit, so as to support more read-write operations.

The effect of instruction set customization on performance and memory utilization can be understood from Figure 3. As we customize instruction set from 32-bit data types to 8-bit types, we are able to effectively improve memory utilization and the overall performance.

Martin at el [13] proposed reconfigurable processor architecture for mobile phones. Dynamically Configurable System on Chip (CSoC) architecture has been optimized for mobile communications. CSoC's are customized for a specific application and its architecture consists of processor core, memory, ASIC cores, and on-chip reconfigurable hardware units.

Most of the smart phones today are single or dual-core SoC's. For most of the mobile applications, faster dual-core CPU provides better performance than quad-core SoC's. Future SoC's for mobile will become more sophisticated improving the overall performance.

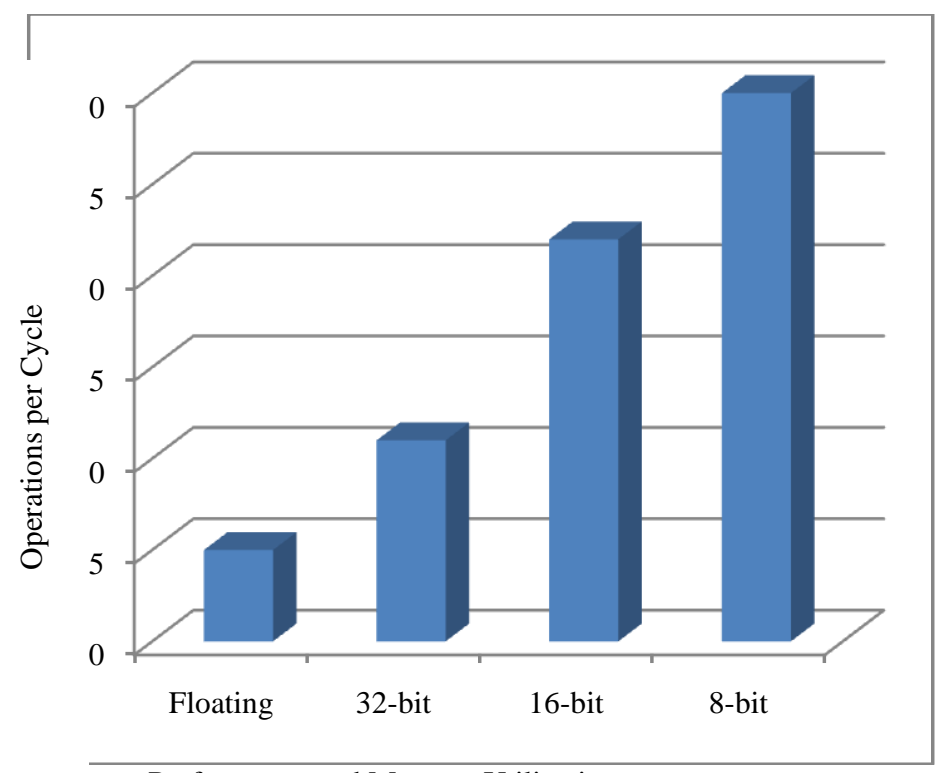

Performance and Memory Utilization

Figure 3: Effect of Instruction Set Customization on performance and memory utilization

\subsection{ARM Processors for Mobiles}

ARM based processors are the most widely used in modern Smart phones. ARM is a32-bit instruction set architecture based on RISC architecture [10]. ARM processors are particularly used in Smart phones because of its low power consumption and great performance.

ARM holdings provide chip design and instruction set customization licenses to third party vendors like Apple, Qualcomm etc. who design their own products based on the provided architecture.

Various ARM architectures used in Smartphone are ARMv5 utilized in low-end devices, and ARMv6, ARMv7 utilized in recent high performance devices. ARMv7 includes a hardware floating-point unit (FPU) providing improved speed. The 32-bit ARM architecture, such as ARMv7-A, is the most extensively used architecture in mobile devices.

ARM architecture is the main hardware architecture for most of the operating systems of mobile devices such as iOS, Android, Windows Phone, Windows RT, Bada, Blackberry OS/Blackberry10, MeeGo, Firefox OS, Tizen, Ubuntu Touch, Sailfish and Igelle OS.

\section{COMPARATIVE STUDY OF CONTEMPORARY MOBILE PHONE PROCESSORS}

ARM Cortex, Snapdragon, Nvidia Tegra are among the most widely used mobile processors.

\subsection{ARM Cortex Processors}

ARM Cortex processors cores are categorized into the following variants:

$>$ Cortex-A Processors (ARM Application Processors)

$>$ Cortex-R Processors (ARM Embedded Real-time Processors)

$>$ Cortex-M Processors (ARM Embedded Processors) 


\section{DecureCore Processors (ARM Secure Processors)}

As an example, consider the architecture of ARM Cortex-A8 depicted in Figure 4. This architecture is based on NEON SIMD media and signal processing technology for providing audio, video, and 3D graphics to mobile applications. Instruction ser architecture of ARM Cortex-A8 implements Thumb-2 instruction set encoding consisting of 16-bit long instructions which require less external memory. AMBA (Advanced Microcontroller Bus Architecture) bus interface supports input and output data buses that are either 64 or 128bit wider. It performs L2 cache fills and non-cacheable accesses for both instructions and data.

Figure 4: ARM Cortex-A8 Architecture

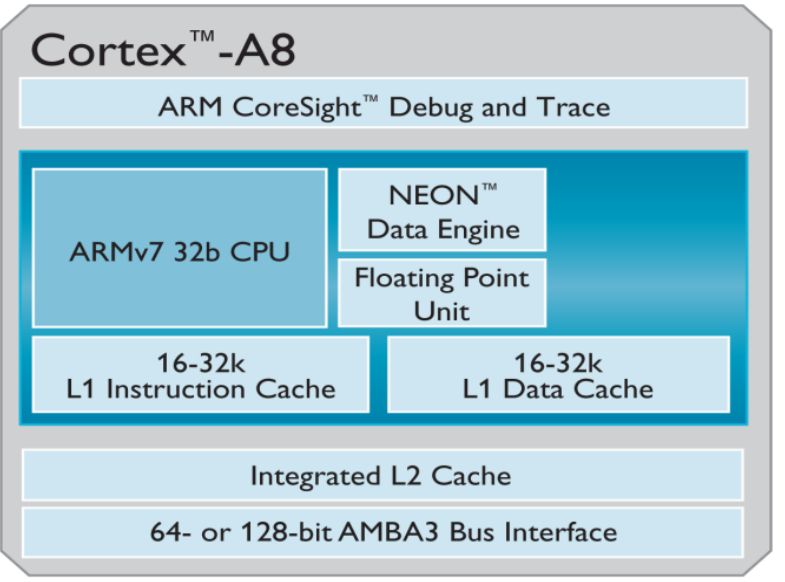

A single core ARM Cortex A8 processor with $1.4 \mathrm{GHz}$ clock speed was considered reasonably enough till 2011. In the year 2014, ARM processors clocked at $3 \mathrm{GHz}$ will become a reality. These upcoming $20 \mathrm{~nm}$ manufactured processors are expected to offer $25 \%$ less power consumption and will allow up to $30 \%$ faster clock speeds.

Mobile computing is gearing up for a drastic change this year with the advent of 64-bit ARM based processors which are expected to provide up to $50 \%$ performance improvement over existing 32-bit ARM processors. ARM's new CortexA50 processor series based on the ARMv8 architecture includes the Cortex-A53 and Cortex-A57 processors. The Cortex-A57 is a performance-oriented applications processor, while the Cortex-A53 is a power-efficient application processor.

In the near future, Android 5.0 will efficiently utilize the new ARMv8 64-bit architecture. Apart from the proposed 64-bit architectures, upcoming mobile phones will be equipped with $4 \mathrm{~GB}$ of RAM to support increasing complexity of 3D games on Android.

\subsection{Qualcomm Snapdragon Processors}

Snapdragon is a family of mobile system on a chip (SoC) processor architecture provided by Qualcomm. Scorpion, the original snapdragon CPU had many features similar to ARM Cortex-A8 core based on ARMv7 instruction set, but with an added advantage of higher performance utilizing SIMD operations.

Qualcomm Snapdragon Soc's are build around Krait processor architecture, shown in Figure 5. It integrates LTE (Long Term Evolution) modem to support seamless connectivity across $2 \mathrm{G}, 3 \mathrm{G}$ and $4 \mathrm{G}$ LTE networks. This architecture supports a wider front-end, with the ability to fetch and decode three instructions per clock. Adreno GPU in this architecture delivers improved advanced graphics performance. With Hexagon DSP's, this architecture provides low power operation for a variety of multimedia applications like enhanced audio/video.

Figure 5: Qualcomm Snapdragon Processor Architecture

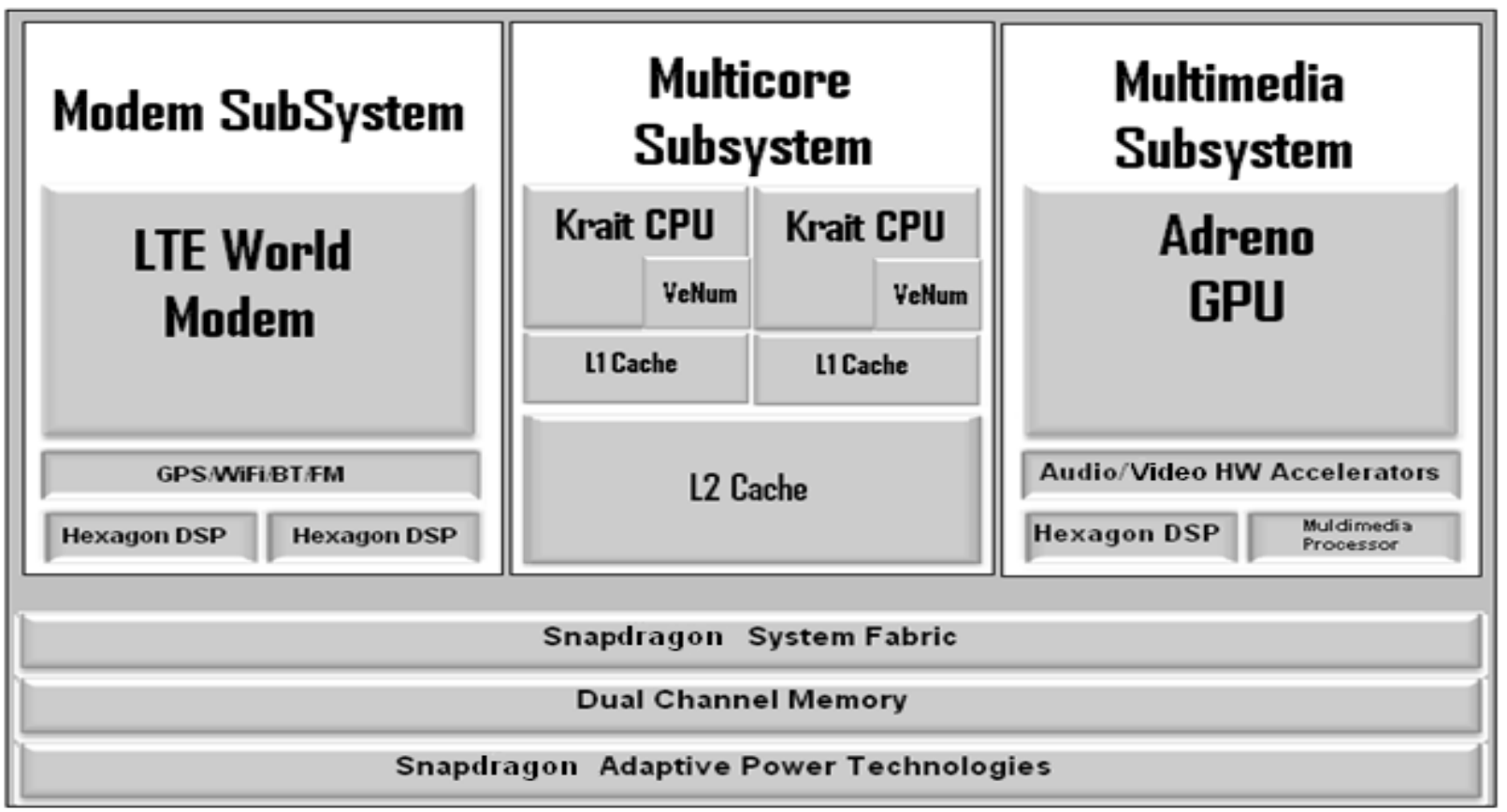

Qualcomm was one of the first to introduce a $28 \mathrm{~nm}$ processor in 2012 with its Snapdragon S4 series processor. GPU and overall architecture are refined inside the Snapdragon 600 and 800 , but they are still utilizing $28 \mathrm{~nm}$ processors.
In the year 2013, Qualcomm Snapdragon 800 processor with Krait $400 \mathrm{CPU}$ cores providing $2.3 \mathrm{GHz}$ clock speed outperformed all other processors in the mobile segment. 
As shown in figure 6 below, Snapdragon 800 processor consists of $28 \mathrm{~nm} \mathrm{HPm}$ quad core Krait $400 \mathrm{CPU}$ for high performance, Adreno 330 GPU for improved graphics performance, Hexagon DSP for low power operation, and Gobi $^{\text {TM }}$ True 4G LTE modem for connectivity.

Figure 6: Qualcomm Snapdragon 800 Processor Architecture

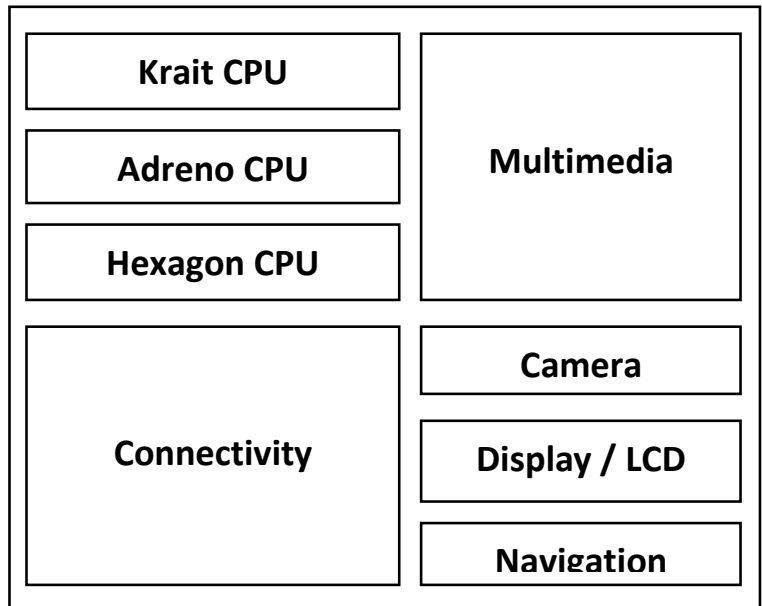

Snapdragon 800 processors are designed to enable incredibly fast apps and web browsing, visually stunning graphics, breakthrough multimedia capabilities, seamless communications virtually anytime, anywhere, and outstanding battery life for premium smart phones.

Snapdragon 800 processor provides seamless connected computing and a rich mobile experience with advanced features like Ultra HD video, multichannel HD audio and

\section{advanced imaging.}

\subsection{Nvidia Tegra Processors}

Tegra is a SoC series for mobile devices developed by Nvidia. It integrates $\mathrm{ARM}$ architecture $\mathrm{CPU}$, graphics processing unit (GPU), memory controllers, etc. on a single package. It enables high performance and low power consumption for audio/ video applications.

Nvidia Tegra 4 processor is a quad-core Soc with increased GPU cores, faster clock cycles, and improved efficiency. The GPU architecture of Tegra 4 is shown in Figure 6. Vertices are processed by six VPE (vertex processing engine) units. Next, vertices are cached by IDX unit. Vertices are then passed to raster engine, which produces pixel fragments. Early-Z unit tests pixel fragments for Z-depth and passes only visible pixels. Early-Z processing in Tegra 4 GPU architecture results in improved performance and power savings. GPU includes four pixel fragment shader pipes which implements VLIW architecture. Each pixel shader unit also contains a texture filtering unit, with their own L1 and L2 cache.

According to an analysis of upcoming ARM processors, it was revealed that Nvidia's Tegra 4 SoC beats the best Qualcomm Snapdragon processor in terms of performance. Though Nvidia have designed a technically faster SoC, but when it comes to power efficiency Qualcomm Snapdragon processors have an edge.

Nvidia has recently launched next generation mobile processor, Tegra K1. It is a mobile processor with 192 graphics cores for mobile gaming applications. Nvidia K1 was launched with a support for two versions: traditional 32-bit "4+1" ARM cores like Tegra 4, and dual core 64-bit version. It is said that Tegra K1 is even more powerful than either the Xbox 360 or the PlayStation 3

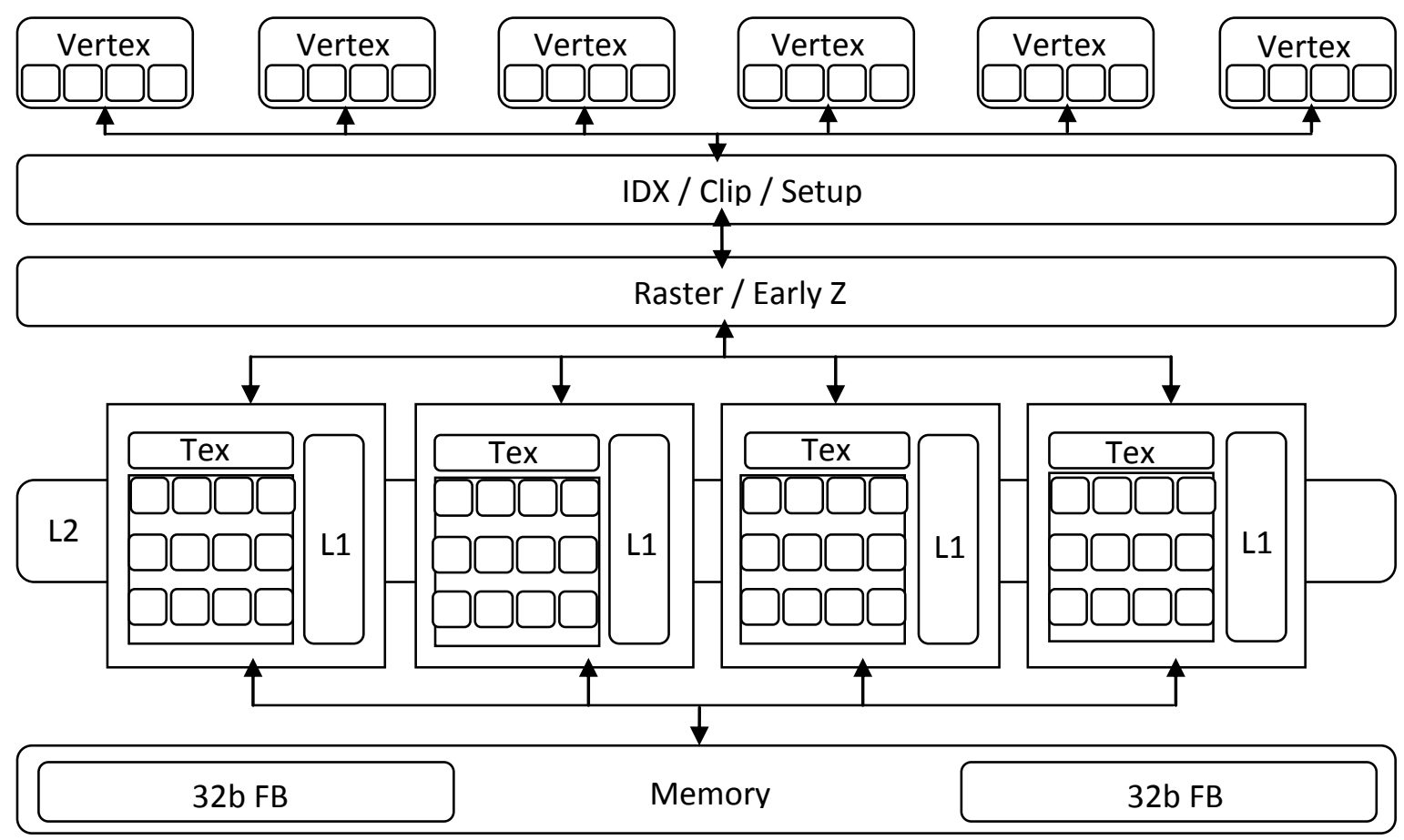

Figure 7: Nvidia Tegra 4 GPU Architecture 


\section{CONCLUSION}

Different vendors are working towards the development of more power efficient mobile processor architectures by looking at the future of mobile computing. All the modern mobile processors are basically ARM-based, designated with fancy names by different cellular companies.

With newer versions of mobile CPU's we will have more powerful Smartphone with new GPU cores, memory interfaces, and many more advanced features. Future mobile SoC's will explore next generation processor architecture to improve the device performance. Mobile processing unit manufacturers are working hard to develop powerful cell phone devices.

To support next-generation data-centric mobile devices, processor architecture has to be designed considering new approaches. Still, the development in mobile processors is driven by factors such as low-power consumption, user interface performance, time to market, etc.

\section{REFERENCES}

[1] Farinaz Kaushanfar, Vandana Prabhu, Miodrag Potkonjak, Jan M. Rabaey: "Processors for Mobile Applications".

[2] Dr. Margarita Esponda: "Trends in Hardware Architecture for Mobile Devices".

[3] Shiv Chaturvedi: "The role of digital signal processors (DSP) for 3G mobile communication systems", International Journal on Emerging Technologies 1(1): 23-26(2010).

[4] "TMS320C62xx CPU and Instruction Set Reference Guide": Texas Instruments.
[5] Matthias H. Weiss, Frank Engel, and Gerhard P. Fettweis: "A new Scalable DSP Architecture for System on Chip (SOC) Domains", IEEE International Conference on Acoustics, Speech, and Signal Processing.

[6] Alan Gatherer, Trudy Stetzler, Mike McMahan, and Edgar Auslander, Texas Instruments: "DSP-Based Architectures for Mobile Communications: Past, Present and Future".

[7] Ravi Managuli, Yongmin Kim: "VLIW Processor Architectures and Algorithm Mappings for DSP Applications".

[8] VLIW Architectures for DSP: "Berkeley Design Technology, Inc.”

[9] Andrew Fallows and Patrick Ganson: "Smartphone Hardware Architecture".

[10] Leonid Ryzhyk: "The ARM Architecture".

[11] “ARM Processor Architecture”, SOC Consortium.

[12] Georgescu, M.D: "Evolution of Mobile Processors", Communications, Computers and signal Processing, 2003. PACRIM. 2003 IEEE Pacific Rim Conference.

[13] Martin Vorbach, Gurgen Becker: "Reconfigurable processor architectures for mobile phones", Parallel and Distributed Processing Symposium, 2003. Proceedings. International.

[14] Russel Tessier and Wayne Burleson: "Reconfigurable Computing for Digital Signal Processing", Journal of VLSI Signal Processing 28, 7-27, 2001. 